\title{
Evaluating the Effectiveness of Targeted Public Health Control Strategies for Chlamydia Transmission in Omaha, Nebraska: A Mathematical Modeling Approach
}

\author{
K. M. Monirul Islam ${ }^{*}$, Ozgur M. Araz ${ }^{2}$ \\ ${ }^{1}$ Department of Epidemiology, Social \& Behavioral Health College of Public Health, University of Nebraska \\ Medical Center, Omaha, USA \\ ${ }^{2}$ Department of Health Promotion, Social \& Behavioral Health College of Public Health, University of Nebraska \\ Medical Center, Omaha, USA \\ Email: ${ }^{*}$ kmislam@unmc.edu
}

Received 20 June 2014; revised 20 July 2014; accepted 10 August 2014

Copyright (C) 2014 by authors and Scientific Research Publishing Inc.

This work is licensed under the Creative Commons Attribution International License (CC BY). http://creativecommons.org/licenses/by/4.0/

(c) () Open Access

\begin{abstract}
Objectives: Sexually Transmitted Infections (STIs) have a great public health impact globally. STIs are one of the most critical health problems in the United States of America (USA). Here, we present a mathematical model for testing several interventions that are designed for various communities in order to control the Chlamydia epidemic. Study Design: Based on a community sexual behavior survey, we constructed and parameterized a mathematical disease transmission model to estimate the spread dynamics of Chlamydia in young adults in the northern part of Omaha, Nebraska. Methods: A differential equations based continuous time simulation model is run for various scenarios. The model considers only one age group i.e., 19 - 25 ages, which is considered as the highest risk group for this sexually transmitted disease. Our model assumes homogeneous mixing within this age group and use published estimates to model mixing rates between individuals. Results: The presented model quantified the potential value of screening and treatment programs for Chlamydia in reducing the burden of disease in this specific community. By increasing the screening and treatment rates from $35 \%$ to $85 \%$, great public health benefit can be achieved in two years, i.e., total cases reduction around $9 \%$ just in this considered age group. Conclusions: Computational results show that behavioral change based interventions on prevention have some effect on reducing the prevalence in the targeted age group; however, more benefit can be obtained with frequent screening and treatment programs.
\end{abstract}

\footnotetext{
"Corresponding author.
}

How to cite this paper: Islam, K.M.M. and Araz, O.M. (2014) Evaluating the Effectiveness of Targeted Public Health Control Strategies for Chlamydia Transmission in Omaha, Nebraska: A Mathematical Modeling Approach. Advances in Infectious Diseases, 4, 142-151. http://dx.doi.org/10.4236/aid.2014.43021 


\section{Keywords}

\section{Chlamydia, Mathematical Modeling, Screening, Public Health, Policy}

\section{Introduction}

Sexually Transmitted Infections (STIs) have a great public health impact globally. STIs are one of the most critical health problems that the United States of America (USA) is facing currently. Centers for Disease Control and Prevention (CDC) estimated that 19 million new STIs occur every year in the United States [1]. STIs also pose a serious economic burden on the health care system in the country (i.e., \$17 billion annually) [1] and each of these infections is a potential threat to an individual's immediate and long-term health and well-being. Chlamydia, one of the most commonly seen STIs, can cause serious short and long-term problems on an individual's reproductive functions, especially in women, and other health consequences including pelvic inflammatory disease (PID). PID can cause permanent damage to fallopian tubes, uterus, and surrounding tissues, which may lead to infertility, ectopic pregnancy, or premature delivery [2]. Furthermore, individuals infected with chlamydia are at higher risk for HIV infection [3].

In 2011, over 1.4 million cases of chlamydia were reported [1]. However, many cases of chlamydia, like other STIs, go undetected and under reported. CDC estimates 2.8 million chlamydia infections occuring annually in the US [1]. Nationally, the number of chlamydia cases continued to rise since 1991, and a dramatic increase of $8 \%$ was reported in both 2010 and 2011 [1]. When the geographic distribution of cases is analyzed, it is clear that they are clustered in certain neighborhoods where significant health disparities exist. Therefore, the public health interventions for controlling this STI should target specific neighborhoods in order to generate the greatest impact possible. In this paper, we present a mathematical model of Chlamydia transmission that is parameterized by community specific values obtained from a survey based study in North Omaha, Nebraska, a state in the Midwestern Region of the United States. Then, community based intervention strategies are evaluated through this model in terms of their effectiveness of controlling the chlamydia cases in this specific community.

\section{Chlamydia in Douglas County}

In Douglas County, in which Omaha is the largest city, more than $70 \%$ of chlamydia cases are among adolescents (14 - 19 years old) and young adults (20 - 25 years old) [4]-[6]. African Americans are disproportionately affected; $44 \%$ of chlamydia cases and 63\% of gonorrhea cases are African American [4]-[6] though they make up only $12 \%$ of the county's population and $41 \%$ of North Omaha's population [7]. Due to the consistent higher rates reported for chlamydia and gonorrhea compared to the national rates, in spite of many interventions, the Douglas County Health Department declared an epidemic of STIs in 2004. This prompted a study to estimate the prevalence and to identify local risk factors of chlamydia in order to institute more effective public health interventions.

\section{Literature Review}

As we have indicated above, the chlamydia epidemic is a serious public health problem in the US, and especially in communities where significant health disparities exist in relation to socio-economic and demographic factors. Therefore, public health strategies should be evaluated in terms of their effectiveness to optimally use limited public resources before they are implemented in the community. Mathematical modeling and simulation based analyses are invaluable tools for informing public health decision makers and designing a set of possible interventions [8]-[13]. Here we present a summary of mathematical model based interventions designed for various communities in order to control chlamydia epidemic all around the world.

Althaus et al. [8] used mathematical and computational models for evaluating the impact of screening programs in reducing the prevalence of Chlamydia trachomatis. They constructed the SEIRS (Susceptible-ExposedInfected-Recovered-Susceptible) model and used published data to parameterize it. Owusu-Edusei et al. [14] recently used a similar but more complicated version of the model in order to evaluate the effectiveness of chlamydia screening and treatment in jails. Another compartmental model was presented in Regan et al. [15]. 
The model was used to quantify the effectiveness of the annual chlamydia screening program in Australia over 10 years. Their model included not only biological parameters but also behavioral factors associated with age group differentiation incorporated into the model. A stochastic simulation model was presented by Kretzschmar et al. [16] to inform designing screening programs in Bilthoven, Netherlands.

Other computational models are also developed and analyzed for chlamydia transmission studies. Turner et al. [17] developed a network model for understanding the key epidemiological features of chlamydia transmission dynamics and health care seeking behavior in Britain. Individual based models are also developed and their performances are evaluated by comparing partnership formation and dissolution dynamics. Gray et al. used an individual based model to assess the impact of vaccination for chlamydia infections and transmission. Althaus et al. [18] presented three different models with empirical data and suggested using Gini coefficient to calibrate infectious disease models. Similar comparisons are done in Ong et al. [19] for general infectious disease modeling to demonstrate the difference between classical models and pair formation based individual models.

After identifying the effective screening and treatment programs, one challenge that public health departments face is how to allocate limited resources across multiple programs. A cubic binary optimization model is formulated and solved by Tao et al. [20] for optimally allocated resources for STI control and prevention programs. Townshend and Turner [21] presented a system dynamics model to evaluate the effectiveness of screening programs for chlamydia and Evenden et al. [22] used a similar approach for analyzing the targeted screening strategies to develop more cost effective programs in Britain.

Here we present a compartmental mathematical model to evaluate screening and prevention programs for Chlamydia transmission which is parameterized based on North Omaha population characteristics and also a survey for understand the sexual behavior in a targeted age group.

\section{Methods}

A cross-sectional survey was conducted to estimate the prevalence and identify risk factors associated with chlamydia in the northern region of Omaha. We recruited 310 participants 19 to 25 years old during July, 2011-June, 2012 to participate in this study. We interviewed them for risk factors and sexual behaviors associated with chlamydia using a pre-designed questionnaire. We also collected urine samples from study participants for chlamydia diagnosis. We will utilize the information acquired from this survey of the sexual behavior and risk factors of chlamydia to evaluate parameters within the models of chlamydia transmission. The summary statistics about the survey elements for the study population are presented in Table 1.

\subsection{Mathematical Model}

We used a compartmental disease transmission model to estimate the spread dynamics of chlamydia in young adults of North Omaha. The model considers only one age group 19 - 25 years of age, which is the high risk group for this sexually transmitted disease. We assume homogeneous mixing within this age group and use published estimates to model the transmission rated between individuals in this age group (1.95 person/year). The transmission dynamics starts with an index case proportion in the defined community and i.e., $14.39 \%$ prevalence rate, and then individuals move from one compartment to another as the natural history of disease progress. The considered compartments of the model are presented in Figure 1. Specifically, the model tracks the changing numbers of susceptible $S(t)$, exposed $E(t)$, asymptomatically infectious $I_{a}(t)$, symptomatically infectious $I_{s}(t)$, on treatment $T(t)$, naturally recovered $R_{N}(t)$, recovered by treatment $\mathrm{R}_{T}(t)$. We use this model to evaluate the effectiveness of targeted screening and treatment strategies in order to control the transmission of chlamydia in this specific community. In addition to screening and treatment policies, we also quantitatively evaluate the effectiveness of public health strategies that aims to increase preventive measures in this age group, e.g., condom use. In the model, other natural history related progression parameters are assumed from the previous studies published in the literature and values for these parameters are presented in Table 2. Population specific demographic information is obtained from the census and the initial value for index case proportion in the population is assumed based on the survey study mentioned in the earlier section.

$$
\frac{\mathrm{d} S(t)}{\mathrm{d} t}=-\lambda S\left(I_{a}+I_{s}\right)-\lambda_{T} S T+\eta_{N} R_{N}+\eta_{T} R_{T}
$$


Table 1. Summary statistics about the survey elements for the study population in North Omaha.

\begin{tabular}{|c|c|c|c|c|}
\hline \multirow{2}{*}{ Survey Elements } & \multicolumn{2}{|c|}{ Males } & \multicolumn{2}{|c|}{ Females } \\
\hline & $\mathbf{n}$ & $\%$ & $\mathbf{n}$ & $\%$ \\
\hline \multicolumn{5}{|l|}{ Race } \\
\hline White & 13 & 7.1 & 4 & 3.1 \\
\hline Other & 18 & 9.9 & 23 & 18.0 \\
\hline African American & 151 & 83.0 & 101 & 78.9 \\
\hline \multicolumn{5}{|l|}{ Previous STD Testing } \\
\hline No & 81 & 44.5 & 46 & 35.9 \\
\hline Yes & 101 & 55.5 & 82 & 64.1 \\
\hline \multicolumn{5}{|l|}{ Previous Positive STD Test } \\
\hline No & 155 & 85.2 & 88 & 68.8 \\
\hline Yes & 27 & 14.8 & 40 & 31.2 \\
\hline \multicolumn{5}{|l|}{ Previous STD Treatment } \\
\hline No & 153 & 84.1 & 88 & 68.8 \\
\hline Yes & 29 & 15.9 & 40 & 31.2 \\
\hline \multicolumn{5}{|l|}{ Current STD Symptoms } \\
\hline No & 173 & 96.1 & 124 & 96.9 \\
\hline Yes & 7 & 3.9 & 4 & 3.1 \\
\hline \multicolumn{5}{|l|}{ Condom use during last sexual experience } \\
\hline No & 65 & 36.1 & 63 & 50.8 \\
\hline Yes & 115 & 63.9 & 61 & 49.2 \\
\hline \multicolumn{5}{|l|}{ Using condom during the last sexual experience } \\
\hline No & 65 & 36.1 & 63 & 50.8 \\
\hline Yes & 115 & 63.9 & 61 & 49.2 \\
\hline \multicolumn{5}{|l|}{ Using drugs during the last sexual experience } \\
\hline No & 132 & 75.0 & 112 & 92.6 \\
\hline Yes & 44 & 25.0 & 9 & 7.4 \\
\hline \multicolumn{5}{|l|}{ Planning to use condoms in the next 3 months } \\
\hline No & 24 & 13.6 & 23 & 19.0 \\
\hline Yes & 52 & 86.4 & 98 & 81.0 \\
\hline & \multicolumn{2}{|c|}{ Mean (SD) } & \multicolumn{2}{|c|}{ Mean (SD) } \\
\hline Age & \multicolumn{2}{|c|}{$21(2.1)$} & \multicolumn{2}{|c|}{$21.5(2.1)$} \\
\hline Number of sexual partners during the past 12 months & \multicolumn{2}{|c|}{$4.2(5.9)$} & \multicolumn{2}{|c|}{$1.9(2.9)$} \\
\hline Times having unprotected sex over the past 12 months & \multicolumn{2}{|c|}{$14.3(38.1)$} & \multicolumn{2}{|c|}{$9.5(20.3)$} \\
\hline
\end{tabular}


Table 2. Parameter notations and definitions.

\begin{tabular}{|c|c|c|c|}
\hline & Notations and Definitions of Parameters & Values & Reference \\
\hline$\lambda$ & $\begin{array}{c}\text { Transmission rate, i.e. the rate at which infectious } \\
\text { individuals infect susceptible individuals }\end{array}$ & 1.95 person/year & [8] \\
\hline$\lambda_{T}$ & $\begin{array}{l}\text { Transmission rate on treatment, i.e. the rate at which individuals who are } \\
\text { infectious but on treatment infect susceptible individuals }\end{array}$ & 1.46 person/year & [8] \\
\hline$\gamma_{A}$ & Rate of asymptomatic infectious individuals receive treatment & 0.027 & $\begin{array}{l}\text { Hamilton et al. } \\
\quad(2010)\end{array}$ \\
\hline$\gamma_{S}$ & Rate of symptomatic infectious individuals receive treatment & 0.064 & $\begin{array}{l}\text { Hamilton et al. } \\
\quad \text { (2010) }\end{array}$ \\
\hline$\xi^{A}$ & Rate that exposed individual become asymptomatic infectious & 0 - 28 days & [15] \\
\hline$\xi^{s}$ & Rate that exposed individual become symptomatic infectious & 0 - 28 days & [15] \\
\hline$\eta_{N}$ & Rate of naturally recovered individuals become susceptible again & 30 - 60 days & [15] \\
\hline$\eta_{T}$ & Rate of individuals recovered by treatment become susceptible again & 14 - 28 days & [15] \\
\hline$\theta N$ & Rate of naturally recovery & 300 days & [8] \\
\hline \multirow[t]{3}{*}{$\theta T$} & Rate of recovery with treatment & 5 - 9 days & [8] \\
\hline & North Omaha population (19 - 25 years) & 8,000 & [7] \\
\hline & Current Chlamydia Prevalence Rate & $14.39 \%$ & Survey Data \\
\hline
\end{tabular}

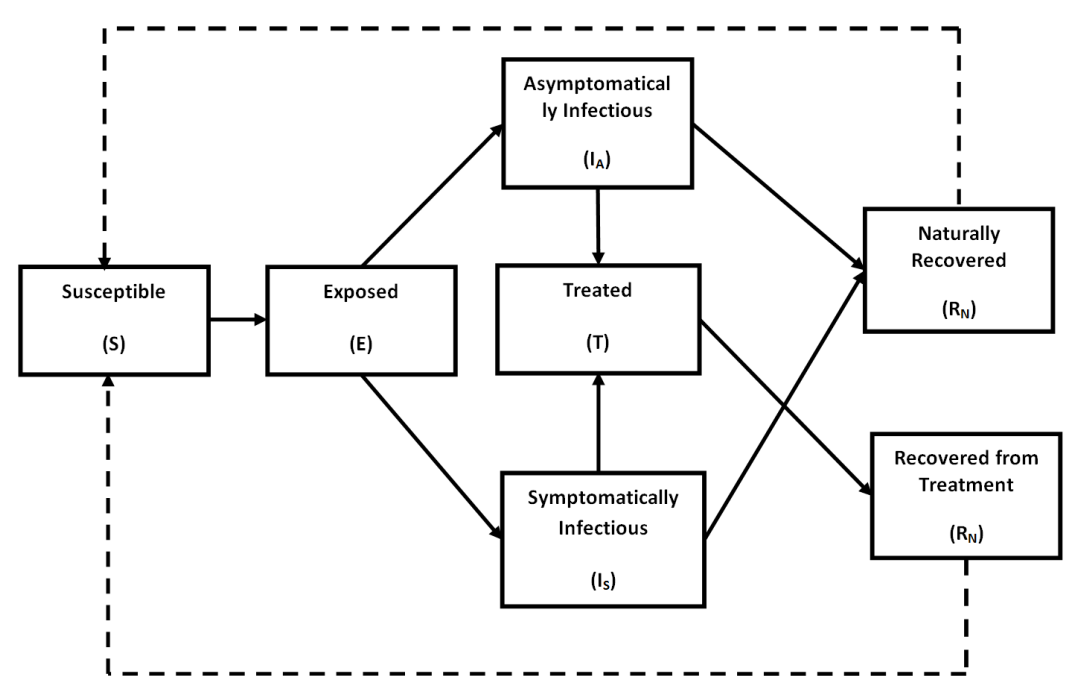

Figure 1. Natural history of chlamydia transmission and progression.

$$
\begin{array}{r}
\frac{\mathrm{d} E(t)}{\mathrm{d} t}=\lambda S\left(I_{a}+I_{s}\right)+\lambda_{T} S T-\left(\xi_{a}+\xi_{s}\right) E \\
\frac{\mathrm{d} I_{A}(t)}{\mathrm{d} t}=\xi_{a} E-\gamma_{a} I_{a}-\theta_{N} R_{N} \\
\frac{\mathrm{d} I_{S}(t)}{\mathrm{d} t}=\xi_{s} E-\gamma_{s} I_{s}-\theta_{N} R_{N} \\
\frac{\mathrm{d} T(t)}{\mathrm{d} t}=\gamma_{a} I_{a}+\gamma_{s} I_{s}-\theta_{T} T \\
\frac{\mathrm{d} R_{N}(t)}{\mathrm{d} t}=\theta_{N} R_{N}\left(I_{a}+I_{s}\right)-\eta_{N} R_{N}
\end{array}
$$




$$
\frac{\mathrm{d} R_{T}(t)}{\mathrm{d} t}=\theta_{T} T-\eta_{T} R_{T}
$$

\subsection{Model Analysis and Results}

In this section we present the simulation results for different screening and treating policies. We have simulated the model for a 10-year period starting from 2013 and presented the results for policies varying from screening and treating for every year to every 20 years. In Figure 2, our results show a substantial reduction in chlamydia cases in the population. Annual screening program in place with $35 \%$ rate is compared to other scheduled screening programs with $45 \%, 55 \%, 65 \%, 75 \%$ and $85 \%$ of hypothetical screening rates for the next 2 years. Since the model dynamics did not change and the system reached to its steady state after 2 years, we do not present simulation results after the end of 2015. By increasing the screening and treatment rates from $35 \%$ to $85 \%$ great public health benefit can be achieved, i.e., reduction in the cases around $9 \%$. These results raised an important question about the comparative effectiveness of the interval of screening program for chlamydia control. The policy makers should allocate resources for an optimum control of STIs in a reasonable time period, which can be achieved with an annual screening program.

In addition to screening and treatment programs, we evaluated the impact of behavioral change interventions on the prevalence rate in the specific age group. The use of a condom for the prevention of chlamydia is also an effective intervention in this population irrespective of either one year or 20 years interval. However, the magnitudes of the case aversion with a behavioral change of condom use remains relatively small even a program can achieve $90 \%$ condom use rate, $0.06 \%$ reduction with a yearly screening program after a year and $0.004 \%$ reduction for the same combination of programs after 3 years (assuming a 50\% base rate of condom use). The result signifies the correct use of a condom every time for prevention of STIs.

Table 3 represents the effects of combined screening for chlamydia screening programs and behavioral change on condom use. Results show that there is an increasing reduction of Chlamydia infections when screen and treat intervention is used in combination with increasing condom use.

\subsection{Analysis on Parameter Sensitivity}

In addition to the analyses presented above for policy evaluation and sensitivity of health outcomes on various implementations of policies, in this section we present sensitivity of outcomes on several parameter values.

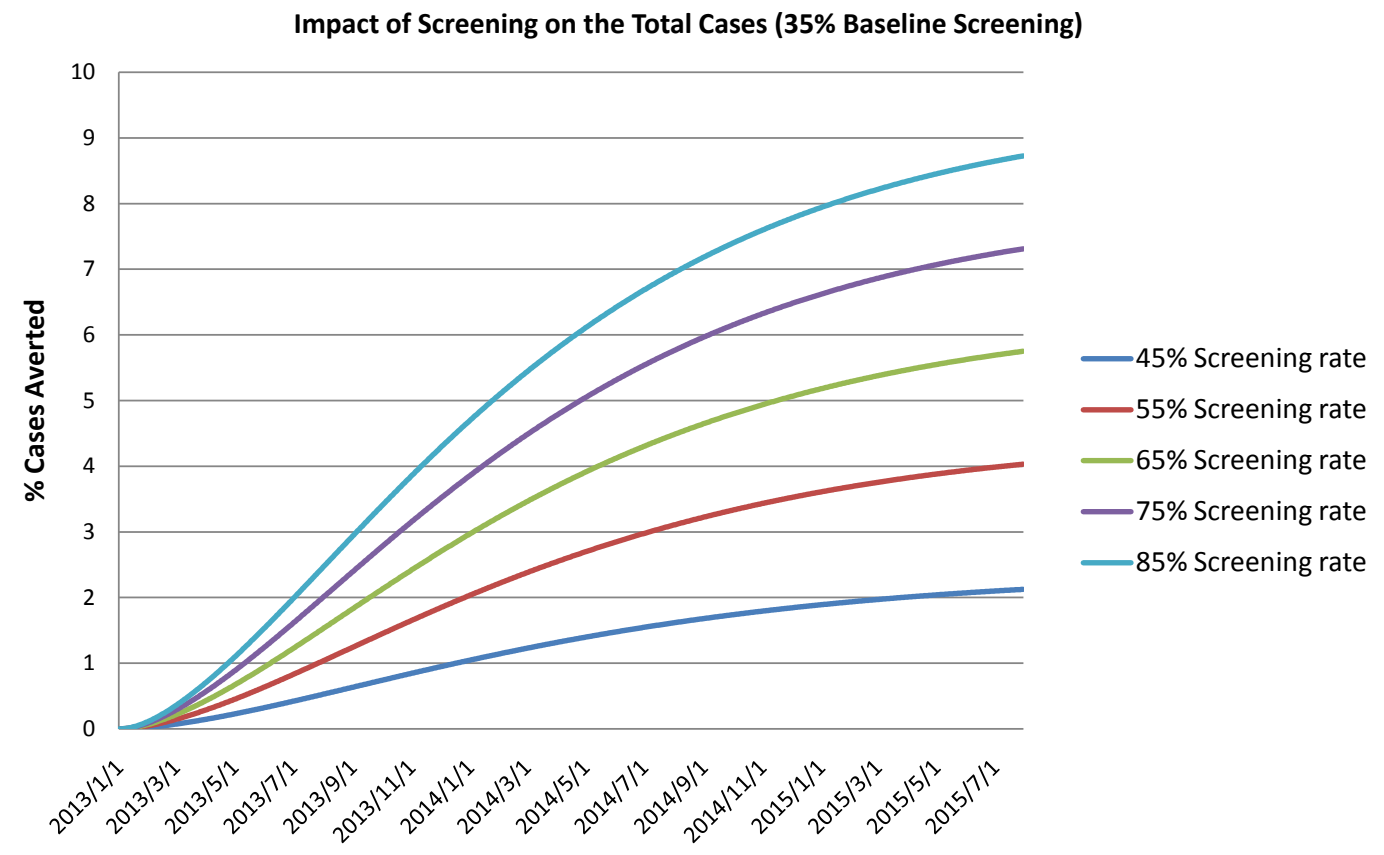

Figure 2. Impact of screening policies and \% cases averted through screening programs. 
Table 3. Prevalence rate and combined effectiveness of screening and behavioral change programs.

\begin{tabular}{|c|c|c|c|c|c|}
\hline \multirow{2}{*}{$\begin{array}{c}\text { Results After } 1 \text { year } \\
\text { Screen + Treat }\end{array}$} & \multicolumn{5}{|c|}{ Behavioral Interventions (Condom Use \%) } \\
\hline & $50 \%$ & $60 \%$ & $70 \%$ & $80 \%$ & $90 \%$ \\
\hline 20 years & 1.75 & 1.72 & 1.7 & 1.67 & 1.64 \\
\hline 10 years & 1.69 & 1.66 & 1.64 & 1.61 & 1.58 \\
\hline 5 years & 1.58 & 1.55 & 1.53 & 1.5 & 1.48 \\
\hline 2 years & 1.28 & 1.26 & 1.24 & 1.22 & 1.2 \\
\hline 1 year & 0.92 & 0.9 & 0.89 & 0.87 & 0.86 \\
\hline Results After 3 years & \multicolumn{5}{|c|}{ Behavioral Interventions (Condom Use \%) } \\
\hline Screen + Treat & $50 \%$ & $60 \%$ & $70 \%$ & $80 \%$ & $90 \%$ \\
\hline 20 years & 0.19 & 0.17 & 0.16 & 0.15 & 0.14 \\
\hline 10 years & 0.16 & 0.15 & 0.14 & 0.13 & 0.12 \\
\hline 5 years & 0.13 & 0.12 & 0.11 & 0.1 & 0.09 \\
\hline 2 years & 0.06 & 0.05 & 0.05 & 0.05 & 0.04 \\
\hline 1 year & 0.016 & 0.015 & 0.014 & 0.013 & 0.012 \\
\hline
\end{tabular}

Since there are great uncertainties on the disease specific parameters, especially due to its asymptomatic nature, the estimates from the previous epidemiological studies were used in our simulations. However, transmission rate and asymptomatic duration can significantly impact the results, and sensitivity analyses on these parameters can inform public health officials for investing more time and time and effort to estimate these parameters. Here we perform sensitivity analyses to determine the impact of uncertainty for transmission rate on the total number cases generated in the sample population. We use Monte Carlo sampling method [23] [24] and run 20 simulations in Powersim software [25], at each time sampling from Normal Distribution with mean 1.95 (i.e., base parameter value used) and variance of 0.95 . In Figure 3, the average of total cases (i.e., total \% of population infected asymptomatically and symptomatically) is presented with 10 percentile and 90 percentile. Although the difference between 10 percentile and 90 percentile of cases were not significant through the early stages of the simulation the gap increases towards the end of the simulation time (i.e., 1825 days), at the end of the simulation the difference is computed around $4.7 \%$.

We also performed sensitivity analysis for the impact of asymptomatic duration on total cases. In the literature there is a significant ambiguity on the estimates used for asymptomatic durations for STIs in general and for chlamydia as well. In our base simulations we used 300 days based on Althaus et al. [8] and again via Monte Carlo sampling method performed the sensitivity analysis. For this parameter we used Uniform distribution with parameters of $(200,400)$ and mean of 300 days. The average for total cases (i.e., rate at which population got infected asymptomatically and symptomatically) with 10 percentile and 90 percentile is presented in Figure 4 . As we can see, the difference between 10 percentile and 90 percentile is more significant, i.e., 17\%, and again, the gap is at its maximum at the end of the simulation time. Since the sensitivity of total cases is much higher on asymptomatic duration than the transmission rate, public health efforts should first focus on obtaining better and more accurate estimates for this parameter in order to inform policy decision making for designing chlamydia prevention interventions.

\section{Conclusions and Discussions}

Our model quantified the potential value of screening and treatment programs for chlamydia in reducing the burden of disease in the North Omaha community. Behavioral change based prevention interventions have some effect on reducing the prevalence in the targeted age group. However, more benefit can be obtained with frequent screening and treatment programs. Cost effectiveness of public health programs should be evaluated by the public health decision makers in order to identify optimal program specific to their communities. 


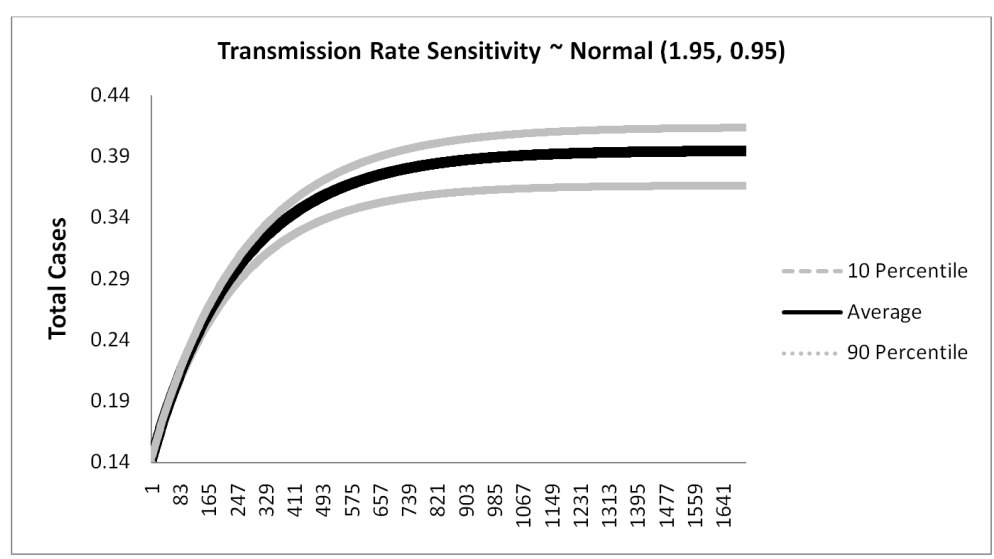

Figure 3. Impact of various screening programs on total number of recovered.

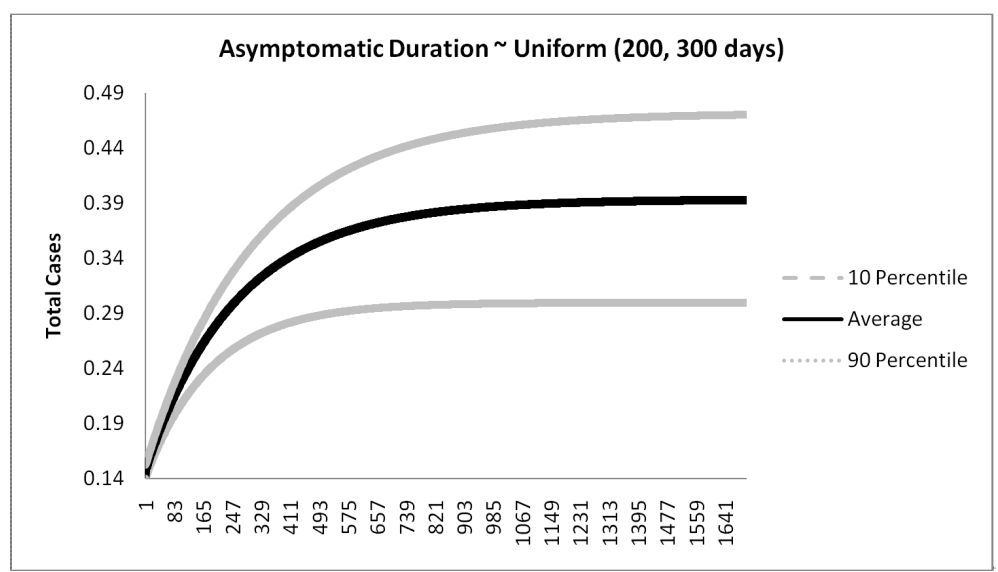

Figure 4. Impact of various screening programs on total number of recovered.

Our mathematical model was designed based on parameters from an epidemiological study conducted in North Omaha, Nebraska and estimates from current literature. We undertook sensitivity analyses to show that the conclusions are robust to parameter assumptions. We have chosen a straightforward model framework, have used conservative assumptions, and considered only the direct effect of screening coverage on prevalence of chlamydia. Our model can easily be modified and adopted in other populations to plan public health programs or examine the effectiveness of screening programs. One limitation of our study is that it does not include any cost effectiveness evaluation for the screening programs; however, previous studies have shown screening and treatment is cost effective. Access to screening should be equitable for all irrespective of socio-economic status, gender, race and ethnicity. However, the additional resources required to increase screening coverage to reach equity among all residents would be more effectively employed in the short term.

Future planning of chlamydia control could include measures to increase screening coverage such as sex and age specific screening paired with or supported by a renewed focus on education. Screening of chlamydia is an effective intervention to reduce sexually transmitted infections and mathematical models can be used to evaluate its effectiveness. Finally, our analyses demonstrate the sensitivity of health outcomes on disease specific parameters. Uncertainty on the asymptomatic duration has significant impact on the total cases that can be generated in the community; therefore, public health research should focus more on obtaining better estimates for this parameter in order to better design optimal intervention strategies.

In the future work, variations on the activity levels, partnership and duration of partnership can be explicitly modeled, rather than assuming instantaneous partnerships. Also, more flexible screening programs can be evaluated, e.g., modeling the behavior of screening for individuals who are in risk groups. Extensive sensitivity 
analyses can also be performed for other parameters in the model.

\section{References}

[1] Centers for Disease Control and Prevention (2012) Sexually Transmitted Disease Surveillance 2011. Centers for Disease Control and Prevention, Atlanta.

[2] Heymann, D.L. (Ed.) (2008) Control of Communicable Diseases Manual. 19th Edition, APHA, Washington DC.

[3] Wasserheit, J.N. (1992) Epidemiological Synergy: Interrelationships between Human Immunodeficiency Virus Infection and Other Sexually Transmitted Diseases. Sexually Transmitted Diseases, 19, 61-77. http://dx.doi.org/10.1097/00007435-199219020-00001

[4] Douglas County Health Department, 2012. http://www.douglascountyhealth.com/images/stories/stats/morbidity/STD2012_short\%20Compatibility\%20Mode.pdf

[5] STDs in Douglas County. Douglas County Health Department, 2012.

[6] Region Population Summary by Race/Ethnicity. Douglas County Health Department, 2011.

[7] U.S. Census Data, 2010. http://www.census.gov/population/www/cen2010/glance/

[8] Althaus, C.L., Heijne, J.C.M., Roellin, A. and Low, N. (2010) Transmission Dynamics of Chlamydia trachomatis Affect the Impact of Screening Programmes. Epidemics, 2, 123-131. http://dx.doi.org/10.1016/j.epidem.2010.04.002

[9] Heijne, J.C.M., Althaus, C.L., Herzog, S.A., Kretzschmar, M. and Low, N. (2011) The Role of Reinfection and Partner Notification in the Efficacy of Chlamydia Screening Programs. The Journal of Infectious Diseases, 203, 372-377.

[10] Garnett, G.P., Mertz, K.J., Finelli, L., Levine, W.C. and St Louis, M.E. (1999) The Transmission Dynamics of Gonorrhoe: Modeling the Reported Behavior of Infected Patients from Newark, New Jersey. Philosophical Transactions of the Royal Society of London. Series B, Biological Sciences, 354, 787-797.

[11] de Vries, R., van Bergen, J.E.A.M., Berh, L.T.W. and Postma, M. (2006) Systemic Screening for Chlamydia trachomatis: Estimating the Cost-Effectiveness Using Dynamic Modeling and Dutch Data. Value in Health, 9, 1-11. http://dx.doi.org/10.1111/j.1524-4733.2006.00075.x

[12] Bansal, S., Grenfell, B.T. and Meyers, L.A. (2007) When Individual Behavior Matters: Homogenous and Network Models in Epidemiology. Journal of the Royal Society Interface, 4, 879-891. http://dx.doi.org/10.1098/rsif.2007.1100

[13] Kretzschmar, M., van Duynhoven, Y.T.H.P. and Severijnen, A.J. (1996) Modeling Prevention Strategies for Gonorrhea and Chlamydia Using Stochastic Network Simulations. American Journal of Epidemiology, 144, 306-317. http://dx.doi.org/10.1093/oxfordjournals.aje.a008926

[14] Owusu-Edusei, K., Gift, T., Chesson, H.W. and Kent, C.K. (2013) Investigating the Potential Public Health Benefit of Jail-Based Screening and Treatment Programs for Chlamydia. American Journal of Epidemiology, 177, 463-473. http://dx.doi.org/10.1093/aje/kws240

[15] Regan, D.G., Wilson, D.P. and Hocking, J.S. (2008) Coverage Is the Key for Effective Screening of Chlamydia trachomatis in Australia. Journal of Infectious Diseases, 198, 349-358. http://dx.doi.org/10.1086/589883

[16] Kretzschmar, M., Welte, R., van den Hoek, A. and Postma, M.J. (2001) Comparative Model-Based Analysis of Screening Programs for Chlamydia trachomatis Infections. American Journal of Epidemiology, 153, 90-101. http://dx.doi.org/10.1093/aje/153.1.90

[17] Turner, K.M.E., Adams, E.J., Gay, N., Ghani, A.C., Mercer, C. and Edmunds, W.J. (2006) Developing a Realistic Sexual Network Model of Chlamydia Transmission in Britain. Theoretical Biology and Medical Modelling, 3, 3. http://dx.doi.org/10.1186/1742-4682-3-3

[18] Althaus, C.L., Turner, K.M.E., Schmid, B.V., Heijne, J.C.M., Kretzschmar, M. and Low, N. (2012) Transmission of Chlamydia trachomatis through Sexual Partnership: A Comparison between Three Individual-Based Models and Empirical Data. Journal of the Royal Society Interface, 9, 136-146. http://dx.doi.org/10.1098/rsif.2011.0131

[19] Ong, J.B.S., Fu, X., Lee, G.K.K. and Chen, M.I. (2012) Comparability of Results from Pair and Classical Model Formulations for Different Sexually Transmitted Infections. PLoS ONE, 7, e39575.

[20] Tao, G., Zhao, K., Gift, T., Qiu, F. and Chen, G. (2012) Using a Resource Allocation Model to Guide Better Local Sexually Transmitted Disease Control and Prevention Programs. Operations Research for Health Care, 1, 23-29. http://dx.doi.org/10.1016/j.orhc.2012.05.001

[21] Townshed, J.R.P. and Turner, H.S. (2000) Analyzing the Effectiveness of Chlamydia Screening. The Journal of the Operational Research Society, 51, 812-824. http://dx.doi.org/10.1057/palgrave.jors.2600978

[22] Evenden, D., Harper, P.R., Brailsford, S.C. and Harinda, V. (2006) Improving the Cost Effectiveness of Chlamydia Screening with Targeted Screening Strategies. Journal of the Operational Research Society, 57, 1400-1412. http://dx.doi.org/10.1057/palgrave.jors.2602134 
[23] Diwekar, U.M. (2003) Introduction to Applied Optimization, Applied Optimization, Vol. 80. Kluwer Academic Publishers, The Netherlands. http://dx.doi.org/10.1007/978-1-4757-3745-5

[24] Araz, O.M., Lant, T., Fowler, J.W. and Jehn, M. (2011) A Simulation Model for Policy Decision Analysis: A Case of Influenza Pandemic on a University Campus. Journal of Simulation, 5, 89-100. http://dx.doi.org/10.1057/jos.2010.6

[25] Powersim Software AS (2003). http://www.powersim.com 
Scientific Research Publishing (SCIRP) is one of the largest Open Access journal publishers. It is currently publishing more than 200 open access, online, peer-reviewed journals covering a wide range of academic disciplines. SCIRP serves the worldwide academic communities and contributes to the progress and application of science with its publication.

Other selected journals from SCIRP are listed as below. Submit your manuscript to us via either submit@scirp.org or Online Submission Portal.
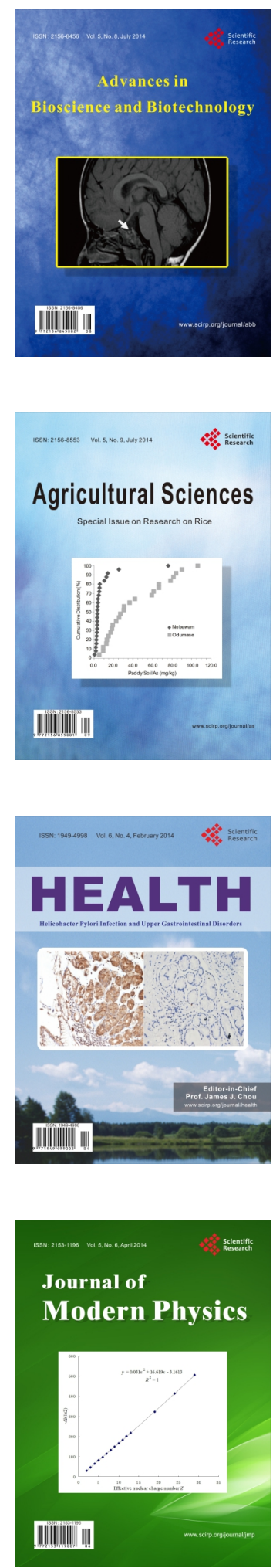
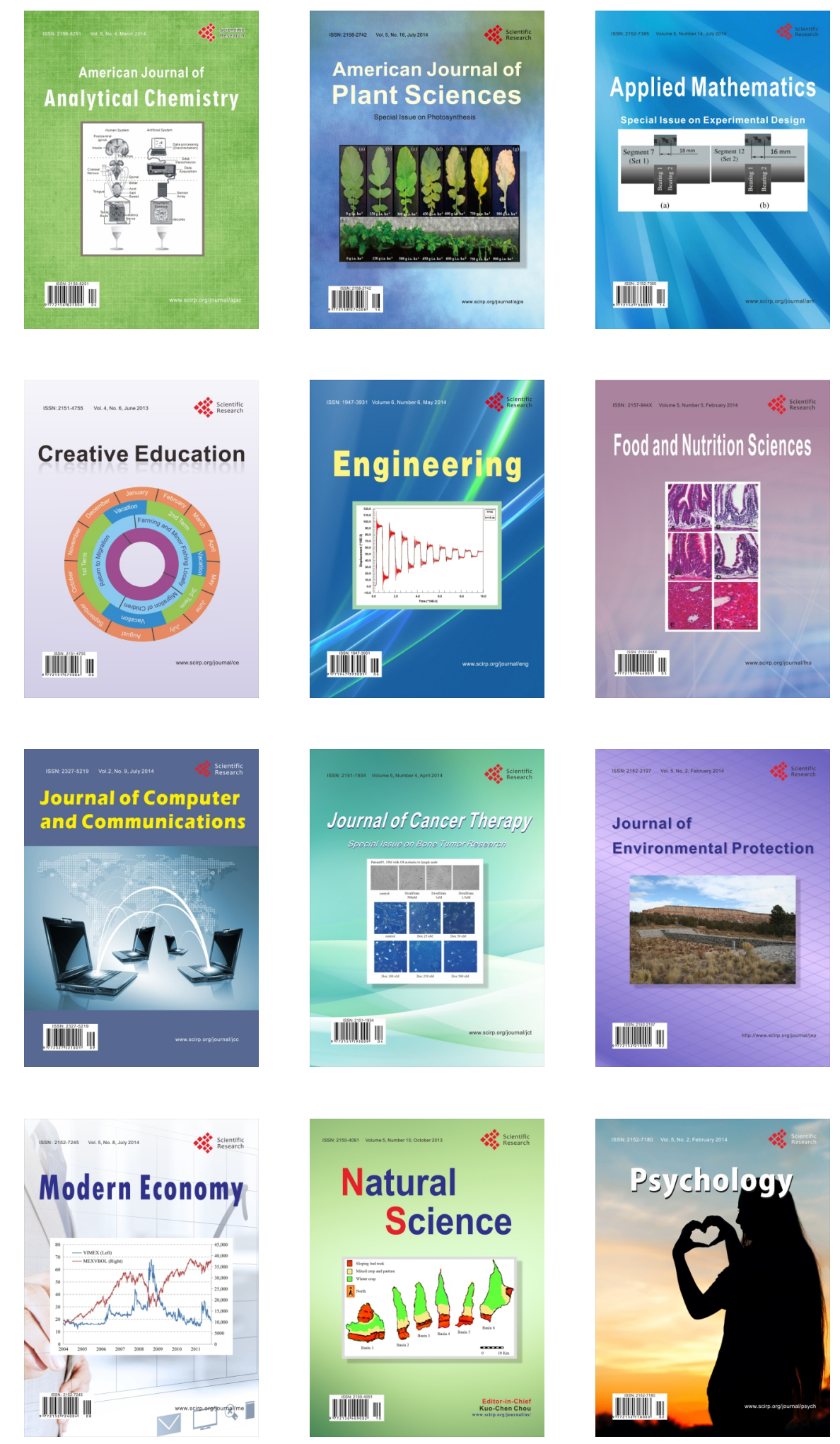